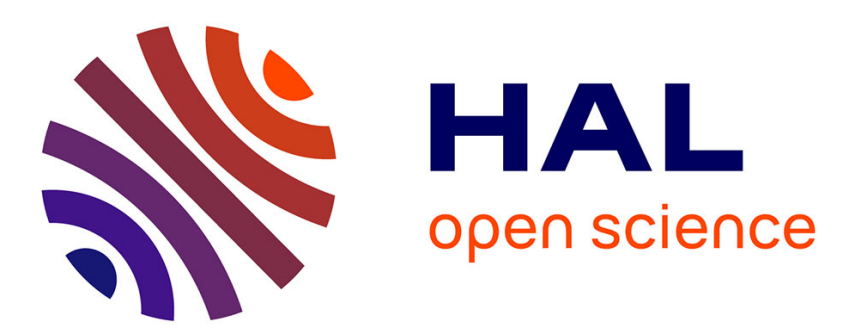

\title{
Dispositif de clivage sous ultra-vide
}

\author{
J.P. Guignard, J. Roussel
}

\section{To cite this version:}

J.P. Guignard, J. Roussel. Dispositif de clivage sous ultra-vide. Revue de Physique Appliquée, 1969, 4 (3), pp.409-411. 10.1051/rphysap:0196900403040900 . jpa-00243304

\section{HAL Id: jpa-00243304 https://hal.science/jpa-00243304}

Submitted on 1 Jan 1969

HAL is a multi-disciplinary open access archive for the deposit and dissemination of scientific research documents, whether they are published or not. The documents may come from teaching and research institutions in France or abroad, or from public or private research centers.
L'archive ouverte pluridisciplinaire HAL, est destinée au dépôt et à la diffusion de documents scientifiques de niveau recherche, publiés ou non, émanant des établissements d'enseignement et de recherche français ou étrangers, des laboratoires publics ou privés. 


\title{
DISPOSITIF DE GLIVAGE SOUS ULTRA-VIDE
}

\author{
Par J. P. GUIGNARD et J. ROUSSEL, \\ Laboratoire de Cristallographie et Physique des Matériaux, Faculté des Sciences, 9I-Orsay.
}

(Reçu le 28 avril 1969.)

Résumé. - Nous décrivons un dispositif répétitif de clivage sous ultra-vide, utilisant la chute d'une bille le long d'une glissière parabolique. Les commandes mécaniques sont effectuées par des bilames dont l'alimentation nécessite seulement des passages électriques.

Abstract. - We describe a device allowing successive cleavages of a single-crystal in an ultra-high vacuum system and using the fall of a metallic ball along a parabolic trajectory. The mechanical controls are done by bimetallic strips and requires only electrical feedthroughs.

Introduction. - Parmi les nombreux paramètres qui déterminent les propriétés des films minces obtenus par dépôt sous vide, la préparation du support joue un rôle déterminant. Le clivage sous vide, en particulier, permet d'obtenir un support cristallin propre.

Dans le cas de l'étude de la croissance épitaxique, l'intérêt de ce mode de clivage est d'autant plus grand qu'il diminue le nombre des marches produites [1], et qu'il favorise l'orientation du film évaporé $[2,3]$.

Conçu pour fonctionner dans un bâti Varian VT 105 dans lequel le vide limite $\left(5 \times 10^{-9}\right.$ torr $)$ est atteint en 12 heures environ, le dispositif que nous avons réalisé devait être répétitif et permettre la réalisation rapide d'une série de 15 à 18 échantillons; une telle

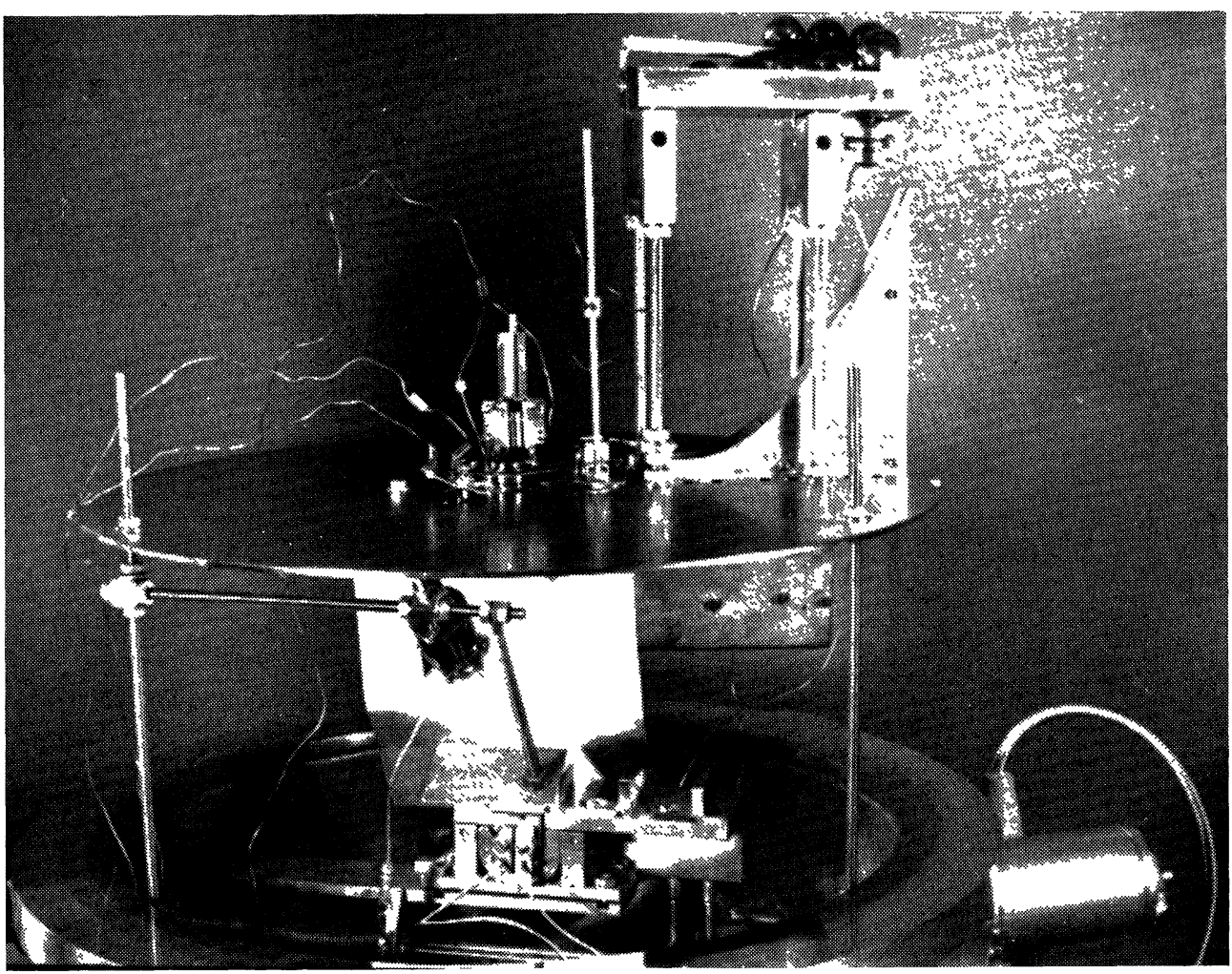

FIG. 1. - Dispositif de clivage sous ultra-vide : vue d'ensemble. 
série, sans remise à l'atmosphère, facilite l'étude de l'influence des gaz résiduels dans l'enceinte [4]. Les difficultés d'étanchéité en ultra-vide et, par conséquent, le coût des passages mécaniques nous ont incités à les éviter au maximum.

La solution adoptée (fig. 1) consiste à provoquer le clivage par la chute d'une bille le long d'une glissière parabolique, les commandes mécaniques étant assurées par les bilames droits ou circulaires. Des passages électriques suffisent à leur alimentation. L'ensemble du système est situé dans l'enceinte.

Description. - Le cristal à cliver a la forme d'un barreau de $1 \mathrm{~cm}^{2}$ de section et de $10 \mathrm{~cm}$ de longueur. Nous avons utilisé du $\mathrm{KCl}$ et du $\mathrm{NaCl}$.

Une bille d'acier de $20 \mathrm{~mm}$ de diamètre, tombant d'une hauteur de $17 \mathrm{~cm}$, en suivant une trajectoire parabolique, choque une lame de rasoir (ou de bistouri), maintenue en contact avec une arête du barreau cristallin. Le barreau est guidé verticalement sur une longueur de $3 \mathrm{~cm}$ par les glissières polies du portecristal. Ces glissières maintiennent le cristal par la pression de ressorts dont l'action peut être annulée par deux bilames.

Le porte-cristal possède deux positions fixes : clivage et évaporation. On passe de l'une à l'autre par une rotation de $90^{\circ}$, commandée par un passage tournant magnétique et une transmission à joints de Cardan. Ge mouvement nécessite un intervalle de l'ordre d'une seconde, temps séparant ainsi le clivage et le début de l'évaporation.

Huit bilames, chauffés par effet Joule, permettent

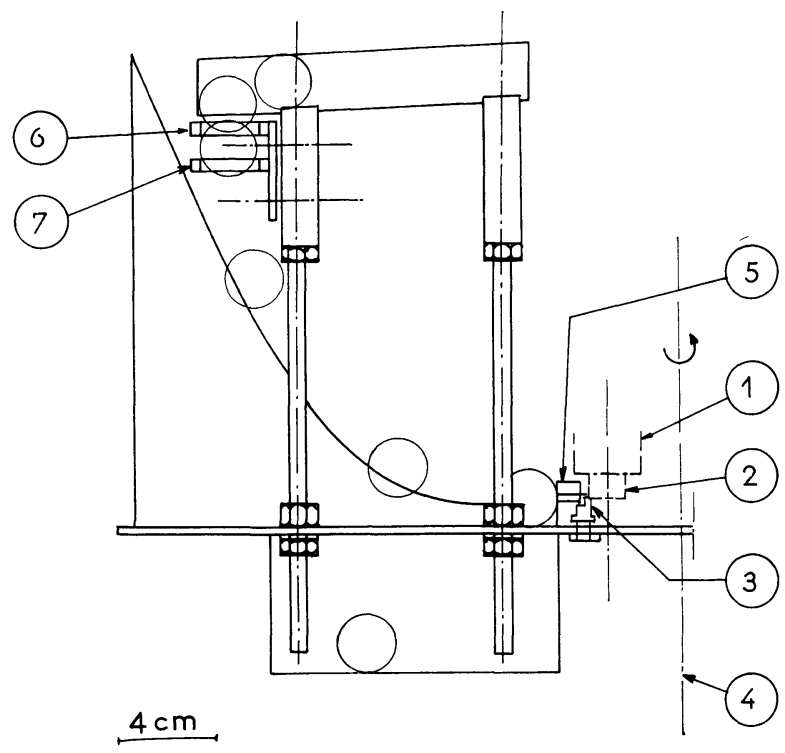

FIG. 2. - Principe de l'appareil : 1, Porte-cristal ; 2 , Cristal à cliver ; 3 , Butée de référence ; 4 , Axe de rotation du porte-cristal ; 5 , Porte-lame ; 6 , Bilame A ; 7, Bilame B. la réalisation d'un cycle au terme duquel est obtenue une lame de $\mathrm{NaCl}$ ou de $\mathrm{KGl}$, de dimensions $10 \times 10$ $\times 2 \mathrm{~mm}$, clivée suivant un plan (001) et supportant le film mince.

Le cycle réalisé est le suivant (fig. 2) :

a) Mise en position clivage par rotation du portecristal;

b) Remise à hauteur du cristal : les bilames du porte-cristal libèrent le barreau cristallin qui descend par gravité jusqu'à la butée de référence;

c) Clivage : chute d'une bille après ouverture successive des bilames $\mathrm{A}$ et $\mathrm{B}$; récupération de l'échantillon obtenu lors du cycle précédent;

d) Mise en position évaporation par rotation de $90^{\circ}$ du porte-cristal;

e) Évaporation : elle a lieu, si besoin est, après le positionnement d'un cache, commandé dans les deux sens de rotation par des bilames spiraux; ce cache permet en particulier la réalisation de marches réfléchissantes destinées à la mesure de l'épaisseur déposée.

Deux améliorations se sont avérées extrêmement utiles en cas de chute accidentelle du barreau cristallin (porte-cristal manœuvré avant le refroidissement total de ses bilames) :

- un bilame permet de remonter ce barreau au-dessus du niveau fixé par la butée de référence,

- un autre bilame permet l'escamotage de cette butée (quelques dixièmes de $\mathrm{mm}$ ) afin d'éviter la détérioration éventuelle, par frottement, du film évaporé.

Les bilames sont alimentés sous faible tension. Le courant est distribué par un sélecteur à 8 positions constitué de trois éléments binaires. Dans l'enceinte, les isolements électriques sont assurés par des bagues en téflon et les conducteurs en cuivre sont réunis par sertissage.

Les bilames ont été fournis gracieusement par la maison Gilby Fodor, dimensionnés suivant les indications du fabricant et formés par nos soins.

Dégazage. - Le dispositif est réalisé en acier inoxydable avec le souci de limiter au maximum la surface totale d'acier et par-là même le dégazage. Tous les filetages ont été saignés transversalement. En fait, la présence de ce dispositif ne modifie en rien les caractéristiques (vide limite et vitesse de pompage) du bâti Varian.

Seul le chauffage du bilame fonctionnant immédiatement avant le clivage pourrait poser un problème de contamination; or, pour une pression initiale de $4 \times 10^{-9}$ torr, lors du déclenchement de la première bille, la pression remonte à $8 \times 10^{-9}$ torr et reprend sa valeur antérieure en une minute environ. Les déclenchements suivants ne modifient pas la pression de plus de $0,2 \times 10^{-9}$ torr dans les mêmes conditions; cette variation sera donc considérée comme négligeable par 


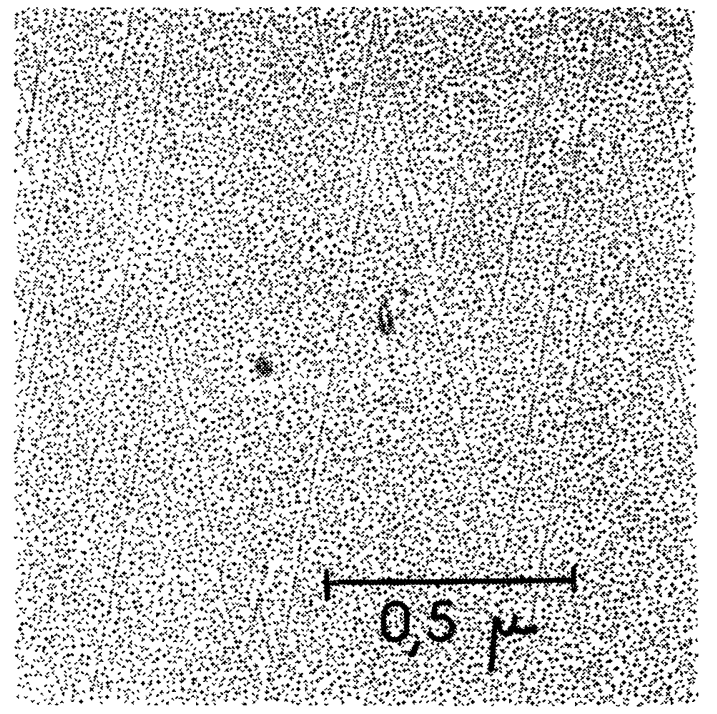

FIG. 3. - Décoration à l'or d'une surface de $\mathrm{NaCl}$ obtenue par clivage sous vide. rapport à celle provoquée par la source d'évaporation.

De par son mode de fonctionnement, ce dispositif n'est évidemment pas étuvable.

Conclusion. - Get appareil de clivage trouve sa justification dans les remarques suivantes :

- parmi les nombreux systèmes proposés, la guillotine [5] et le système de clivage par torsion [2], commandés de l'extérieur de l'enceinte par le déplacement d'une vis ou l'action d'un électroaimant, ne conviennent que pour des échantillons de quelques $\mathrm{mm}^{2}$ de section,

- pour un barreau cristallin de dimensions plus importantes, le choc d'une lame par coup de marteau $[6,7]$ n'assure pas une reproductibilité suffisante [8].

La technique de décoration à l'or des surfaces clivées confirme les observations de Jaunet, Sella et Trillat [1], quant à l'absence de marches sinueuses après un clivage sous vide ( $f$ ig. 3 ).

\section{BIBLIOGRAPHIE}

[1] Jaunet (J.), Selita (C.) et Trilitat (J. J.), C. $R$. Acad. Sci. Paris, 1964, 258, 135.

[2] Ino (S.), Watanabe (D.) et Ogawa (S.), J. Phys, Soc. Japan, 1962, 17, 1074.

[3] Pashleiy (D. W.), Advanc. Phys., 1965, 55, 327.

[4] Roussei, (J.), Guignard (J. P.), Perio (P.) et Okusumi (H.), J. Physique, 1968, 29, 1009.
[5] Gobeli (G. W.) et Alime (F. G.), J. Phys. Chem. Solids, 1960, 14, 23.

[6] Walton (D.), RHodin (T. N.) et Rolims (R. W.), J. Phys. Chem., 1963, 38, 2698.

[7] Gervais (H.), Bull. Soc. franc. minér., 1963, 86, 297.

[8] Volpe (M. L.) et Paschali (C. E.), Rev. Sci. Instrum., 1965, 36, 237. 